\title{
The nuclear translocation of insulin-like growth factor receptor and its significance in cancer cell survival
}

\author{
Shahzaib Chughtai $\odot$
}

Department of Biological Chemistry, University of Michigan, Ann Arbor, Michigan

Correspondence

Shahzaib Chughtai, Department of Biological Chemistry, University of Michigan, $1500 \mathrm{E}$ medical Center Drive, Ann Arbor, MI.

Email: shachu@umich.edu
The nuclear translocation of insulin-like growth factor receptor type 1 (IGF-1R) has been documented in a variety of previous studies. The exact mechanism of this translocation, however, is still poorly understood. Furthermore, the functional role of IGF-1R in the nucleus shows promise of transcriptional control. This function is particularly important in cancer cells. Understanding this role may also give insights into cancer biology and treatment methods. Processes including SUMOylation and clathrin-mediated endocytosis are necessary for IGF-1R nuclear translocation to occur. The antiapoptotic qualities of IGF-1R likely contribute to its function in cancer cells. This review aims to synthesize the work on IGF-1R in order to propose a mechanism of translocation. Using this mechanism, new therapeutic targets can be proposed that hinder the role of IGF-1R in cancer metastasis.

\section{KEYWORDS}

apoptosis, cancer, clathrin-mediated endocytosis, IGF1R, nuclear translocation, SUMOylation

\section{1 | INTRODUCTION}

A growing body of evidence has shown that insulin-like growth factor (IGF) receptor (IGFR) is translocated to the nucleus in cancer cells. IGFR is a transmembrane glycoprotein with a structure that consists of two $\alpha$ subunits and two $\beta$ subunits. ${ }^{1}$ The IGF system has been found to have an important function in the aetiology of breast cancer and in mammary gland function. ${ }^{2}$ This review will investigate the work on the nuclear translocation of IGFR and associated proteins in cancer cells and the potential for new therapeutic targets. Insulin receptor (IR) and IGFR type 1 (IGF-1R) evolved from a common ancestral receptor that functioned in metabolism and organism size regulation. ${ }^{3}$ The two receptors have since taken on different functions, despite having similar structures. Although IR is activated by insulin from the pancreas, IGF-1R uses ligands from the liver. These ligands, IGF-I and IGF-II, are also produced by tumour cells and stromal fibroblasts. As a result, IGF-1R specifically, has shown more potential in cancer research than IR has. Once the ligand binds to IGF-1R, the receptor's tyrosine kinase domains are activated. Subsequently, binding sites for

Current author address: 783 Eagle Avenue, Ann Arbor, MI 48103 docking proteins such as IRS- 1 to IRS- 4 and collagen domain protein are phosphorylated. These proteins trigger the phosphatidylinositol3-kinase and RAS/RAF/mitogen-activated protein kinase signalling pathways. ${ }^{3}$ The 2012 study by Sarfstein et al aimed to investigate the nuclear translocation of IGF-1R and the related IR in breast cancer cells, the effect of IGF-IR and IR levels on IGF-1R biosynthesis in breast cancer cells with oestrogen receptor and without oestrogen receptor, and the role of IGF-1R as a transcription factor in the context of IGF-1R gene regulation. ${ }^{2}$ The authors identified an oestrogen receptor dependent pathway of autoregulation of IGF-IR gene expression by cellular IGF-IR. This gene regulation was mediated at the transcription level. There are three potential outcomes for internalized IGF-1R: It can go to the plasma membrane, it can be degraded, or it can go to the nucleus. A significant amount of IGF-1R has been found in the nuclei of advanced or malignant prostate cancer cells. ${ }^{4}$ While investigating the purpose of IGF-1R in cancer cells, the authors found IGF-1R binding sites at or around the transcription start sites of certain genes. These genes included JUN and FAM21 and involved RNA pol II occupancy and histone marks indicating active enhancers and promoters. This recruitment of IGF-1R to chromatin was inducible. 


\section{RESULTS AND DISCUSSION}

\subsection{InsR and IGF-1R have functional differences}

A minireview by Sarfstein and Werner in 2013 compared the form and function of IR (InsR) and insulin like growth factor 1 receptor (IGF-1R). ${ }^{5}$ They found considerable homologies between the two with $84 \%$ in their tyrosine kinase domains, $45 \%$ to $65 \%$ in their ligand binding domains, and over $50 \%$ in their amino acid sequences. They also found that 12 of the 21 exons in the IGF1R gene are equal in size to homologous exons in the InsR gene. There is an alternative splicing event on exon 11 of InsR that creates two isoforms: InsR-A and Ins-B. InsR-A is mainly expressed in foetal tissues, while InsR-B is mainly expressed in adult liver, muscle, and adipocytes. IGF-1R however is not found significantly in adipose tissue and not at all in the liver. Instead, it is highly expressed in the brain.

\section{2 | SUMOylation is necessary for IGF-1R nuclear translocation}

A number of studies suggest that InsR and IGF-1R are translocated to the nucleus; however, the mechanism for this is unclear. Both receptors are found in the perinuclear and nucleolar areas and are modified by small ubiquitin-like modifier (SUMO). Ligand dependent SUMOylation is likely a necessity for nuclear translocation to occur. The tyrosine kinase domain lysine residues, where SUMOylation occurs, are homologous and conserved across different species. To test the necessary nature of SUMOylation, these sites underwent mutagenesis, which prevented nuclear translocation and associated gene activation. ${ }^{6}$ SUMOylation of IGF-1R has also shown an association with increased cell proliferation. ${ }^{7}$ Lin et al used FACS analysis to compare apoptosis rates of cells with wild type IGF-1R (R-WT) to those with mutated SUMOylation sites (R-puro and R-TSM). Relative numbers of wild type IGF-1R-containing cells remained significantly higher than those of the two mutated groups. They also tested the specific effects of IGF-1R SUMOylation on cell cycle progression. The researchers brought each of their cell lines into G1 phase using 36-hour serum starvation. They found that the most notable impact was the relative increase in $S$ phase cells and decrease in G1 phase cells for the R-WT group. They also looked at the expression of different cyclins, CDKs, and CIP/KIP CDK inhibitors that control the cell cycle phases. The R-WT group uniquely showed an increase in G1/S cyclin D1 after 10 hours, an increase in S phase cyclin A after 16 hours, and an increase in G2/M cyclin B1 after 16 and 24 hours. The researchers concluded that IGF-1R SUMOylation affects cell proliferation by stimulating G1-S progression of the cell cycle. ${ }^{7}$ A study by Deng et al in 2011 showed that IGF-1R does not directly translocate to the nucleus from the endoplasmic reticulum or Golgi apparatus, as opposed to cell surface. ${ }^{8}$ Ligand-mediated phosphorylation of InsR and IGF1R has also been found to be important for their nuclear localization. In the 2010 study by Aleksic et al, the authors used dansylcadaverine and dynamin-1 inhibitor dynasore to inhibit clathrin-mediated IGF-1R endocytosis. ${ }^{1}$ Both significantly prevented nuclear IGF-1R translocation (dansylcadaverine: $P<.001$; dynamin-1 inhibitor dynasore: $P<.05)$. Considering that clathrin is an important player in vesicle formation, this data may suggest that IGF-1R translocates to the nucleus via vesicular transport. This study also investigated whether IGF-1R in the nucleus was cleaved by $\gamma$-secretase, for which it is a known substrate. They concluded that full-length IGF-1R is translocated to the nucleus, rather than being cleaved. To test whether phosphorylation by IGF-1R kinase is necessary for nuclear transport, Aleksic et al used AZ12253801 as a selective inhibitor of the kinase. ${ }^{1}$ They found that pretreatment with this inhibitor resulted in a decrease in phosphorylation and nuclear localization of IGF-1R. IGF-1R was extracted from melanoma cells, grown with and without serum, using immunoprecipitation in a study by Sehat et al and then western blotted using an anti-SUMO-1 antibody. ${ }^{6}$ Their results showed a $245 \mathrm{kD}$ SUMO-1 signal in the presence of serum, indicating that IGF-1R gets SUMOylated. The authors identified Lys ${ }^{1025}$, Lys ${ }^{1100}$, and Lys ${ }^{1120}$ as the three sites of SUMOylation on IGF-1R. When these sites were mutated, IGF-1R nuclear translocation was inhibited, while membranebound IGF-1R remained the same. ${ }^{6}$ In serum starved cells that lacked nuclear IGF-1R, Sehat et al were able to stimulate IGF-1R accumulation in the nucleus using IGF-1. While IGF-1R lacks a nuclear localization sequence (NLS) and does not interact with $\beta$-importin, it can still migrate to the nucleus using the NLS of another protein. ${ }^{9}$ IGF-1 is known to trigger the nuclear translocation of IR substrate 1 (IRS-1), which is associated with IGF-1R in signalling pathways and has an NLS. Sehat et al argue that the potential role of IRS-1 in the transport of IGF-1R to the nucleus should be investigated in future work. ${ }^{6}$

\section{3 | IGF-1R can bind to double stranded DNA}

The ability of nuclear IGF-1R to bind DNA was tested using electrophoretic mobility shift assays with biotin-labelled, double-stranded, 40-base pair oligonucleotides. ${ }^{6}$ Their electrophoretic data showed proteins binding to a DNA probe, along with a supershift in the presence of an IGF-1R antibody (Figure 1). This points to an association between the DNA sequence and IGF-1R, either in complex or by itself. Competitive binding by unlabelled probes diminished the supershift bands, further supporting the notion of a physical interaction between IGF-1R and DNA. Furthermore, the receptor bound to only 4 out of 17 tested DNA probes, indicating that this association is sequence specific. ${ }^{6}$ They tested whether the bound DNA fragments represent regulatory sequences by subcloning them into the pGL4-minimal promoter vector coding for firefly luciferase. Cells that were transfected with IGF-1R-containing plasmids exhibited greater transcription of certain sequences compared with cells with empty vectors. They concluded that the bound DNA sequences are enhancer regions and that IGF-1R acts as a transcriptional activator.

\section{4 | IGF-1R has transcription regulatory capabilities}

A 2012 study by Warsito et al found that IGF-1R binds LEF-1, which is a transcription factor that regulates Wnt signalling and activates 


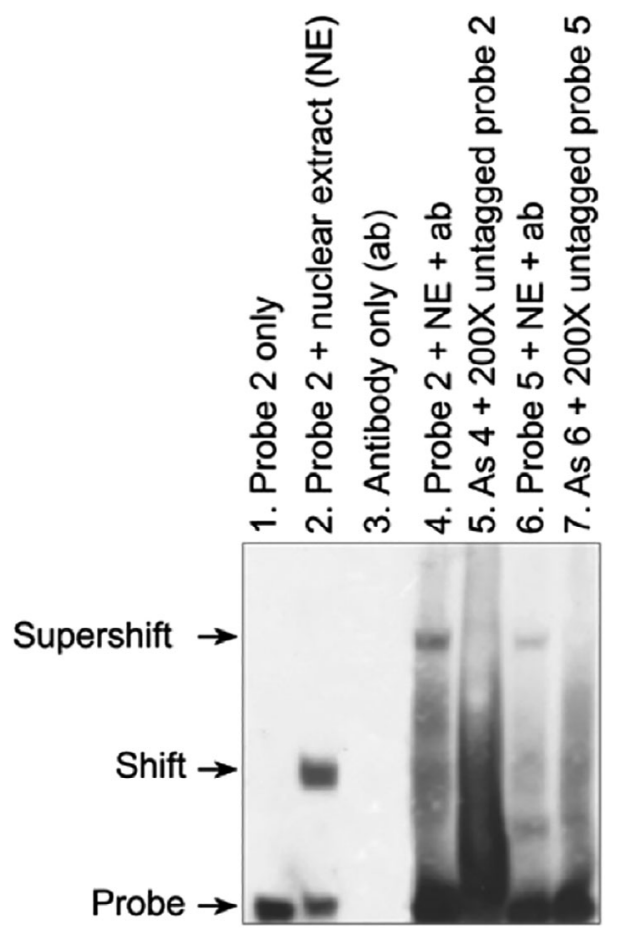

FIGURE 1 EMSA (electrophoretic mobility shift) assay for DNA probes 2 and 5 showing protein binding. Lanes 5 and 7, which lack the supershift, had unlabelled competitive probes at 200 -fold molar mass. This figure is adapted from Sehat et al, $2010^{6}$

LEF-1/TCF targets. ${ }^{10}$ They also saw that nuclear IGF-1R binds to the same site as LEF-1 in the cyclin D1 promoter, which function in cell cycle progression. While IGF-1R can regulate the Wnt cascade, LEF$1 /$ TCF can regulate IGF-1R expression in a bidirectional loop (Figure 2). ${ }^{5}$ Both InsR and IGF-1R translocate to the nucleus of breast cancer cells and were found to bind the IGF-1R gene in cells that were depleted for oestrogen receptors (ERs). ${ }^{2}$ Nuclear InsR was found to diminish IGF-1R gene transcription, whereas nuclear IGF-1R was found to promote its own gene expression. ${ }^{2}$ A 2011 study by Wang et al found that many other tyrosine kinase receptors, including ErbB, fibroblast growth factor, and vascular endothelial growth factor go to the nucleus and function in transcriptional control. ${ }^{11}$ This shows that nuclear translocation and function is a recurring principle in receptor tyrosine kinases.

\section{5 | IGF-1R nuclear activity is prominent in cancer cells}

Many studies on IGF-1R translocation and function in the nucleus focus on cancer cells. The 2012 study by Sarfstein et al observed the ER dependency of IGF-1R autoregulation in human breast cancerderived MCF7 (ER-positive) and MCF7-derived C4.12.5 (ER-depleted) cells. $^{2}$

Inhibition of IGF-1R has been shown to prevent breast cancer metastasis. A dominant negative form of IGF-1R, called 486stop, was injected into a mammary fat pad and did not inhibit tumour growth but did significantly suppress the spread of cancer to the lungs, liver, lymph nodes, and lymph vessels. ${ }^{12}$ A study by Schedlich et al tested IGFR binding proteins (IGFBPs) that are known to help modify the function of IGF. ${ }^{13}$ Nuclear translocation of fluorescently labelled IGFBP-3 and IGFBP-5 was measured in a proportion to rapidly dividing T47D human carcinoma cells. Using this method, IGFBP-5 was shown to translocate to the nucleus of carcinoma cells. The authors defined a sequence within IGFBP-3 basic motif that is needed for its nuclear translocation. The basic carboxyl-terminal domain of IGFBP-3 has a region around amino acids 215 to 232 with notable homology to bipartite NLS. It also shares homology with the DNA-binding domains of several transcription factors. ${ }^{13} \mathrm{Wu}$ et al investigated the role of IGF-1R in the development of prostate cancer and androgen independent disease. ${ }^{14}$ They reviewed the published data on the interactions between IGF-1R and androgen receptor (AR) and suggest that IGF-1R signalling might affect AR compartmentalization. IGF-1R may impact the phosphorylation of $A R$ and thus affect its function. Through its effect on AR activity, IGF-1R can impact prostate cancer development, which supports the possibility of new therapeutic targets. The 2018 study by Aleksic et al found further support for cancer cell nuclear localization by using antibodies for the $\alpha$ and $\beta$ subunits of IGF-1R. ${ }^{4}$ They were able to detect nuclear IGF-1R in highly proliferating primary renal cancer cells, formalin-fixed tumours, preinvasive lesions in the breast, and nonmalignant tissues. ${ }^{4}$ The authors found both $\alpha$ and $\beta$ IGF-1R subunits in the nuclei of cancer cells. IGF-1R is phosphorylated, and then it interacts with chromatin in an IGF induced manner. The authors used this to argue that IGF-1R is directly involved in cancer cell transcription regulation. In addition, they analysed the survival rates of patients with varying degrees of nuclear IGF-1R. They found that higher levels of nuclear IGF1R significantly correlated with shorter survival times. Furthermore, Sarfstein and Werner used the antiapoptotic and pro cell survival capabilities of IGF1R to explain its high expression levels in tumour cells. ${ }^{5}$ IGF-1R activity has been shown to regulate p53 using pathways that can decrease p53 translation or increase p53 stability. ${ }^{15}$ This shows how the receptor may enact its antiapoptotic effects. This would also make sense with the upregulation of IGF-1R that has been observed in advanced stage cancer cells.

\subsection{Summary of IGF-1R regulation mechanism}

Based on the work examined in this review, the mechanism for IGF$1 \mathrm{R}$ nuclear translocation and transcription regulation in cancer cells is as follows: IGF-1R undergoes clathrin-mediated endocytosis and is subsequently SUMOylated on the three 1025, 1100, and 1120 lysine residues. The protein gets phosphorylated by IGF-1R kinase, interacts with IRS-1, and travels to the nucleus. The whole receptor is deposited inside, where it binds to specific genes, including its own, and promotes IGF-1R expression. Nuclear IGF-1R binds to LEF1 transcription factor and upregulates cyclin D1 and axin2 levels. SUMOylated IGF-1R promotes cell proliferation by promoting G1-S cell cycle 

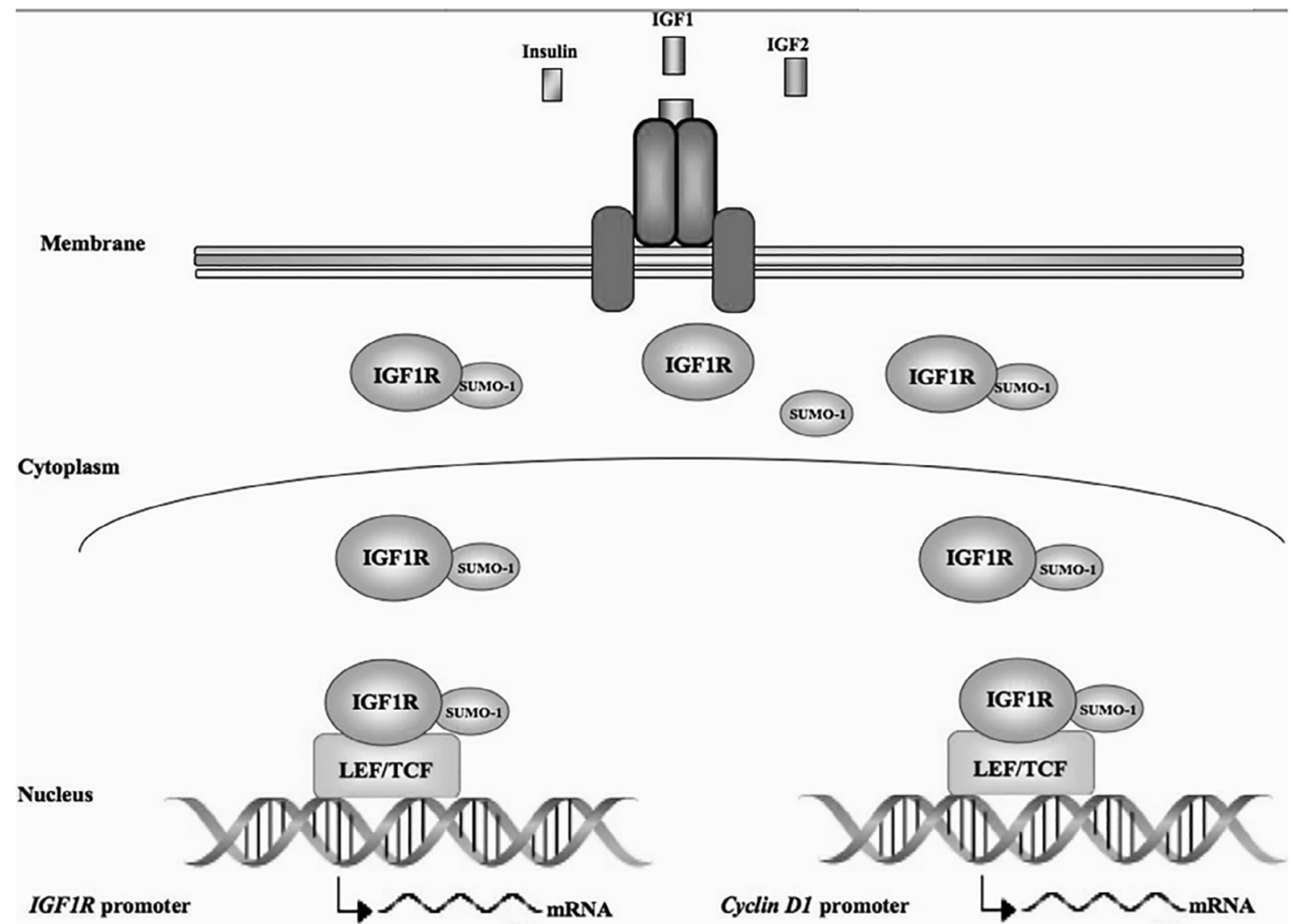

FIGURE 2 The pathway of insulin-like growth factor receptor (IGF-1R) in being SUMOylated, travelling to the nucleus, and regulating gene expression for itself and for Cyclin D1. This figure is adapted from Sarfstein and Werner, $2013^{5}$

progression. Through its disruption of p53 dependent and independent cell death, IGF-1R promotes cancer cell survival and metastasis.

\subsection{Future work}

Using the mechanism of IGF-1R nuclear translocation and transcription regulation, we can identify new targets along the path that can inhibit the role of IGF-1R in cancer cells. The therapeutic potential of IGF-1R for cancer has been controversial, but by targeting different points in the mechanism, a confident treatment method may be developed that can curb metastasis. Such targets could include preventing the SUMOylation process needed for nuclear translocation, inhibiting the clathrin-mediated vesicle formation, and attacking IRS-1 or the associated IGF-1 proteins. By focusing on the nuclear translocation of IGF-1R, we can address its cancer-related effects, while still allowing it to perform its important functions in the cytosol. How exactly SUMOylated IGF-1R stimulates G1-S progression should be further investigated. It may specifically upregulate cyclins D1, A, and B1 while leaving proteins associated with other cell cycle phases unaffected. The role of IRS-1 should be studied in greater detail. It is also important to determine how IGF-1R exactly goes inside the nucleus and the specific involvement of nuclear pores and vesicles. In addition, a cinematographic study to observe the exact paths that IGF-1R takes and which exact areas it is expressed in over the course of this whole process would be helpful in determining the exact mechanism.

\section{DATA AVAILABILITY STATEMENT}

Data sharing is not applicable to this article as no new data were created or analyzed in this study.

ORCID

Shahzaib Chughtai (D) https://orcid.org/0000-0002-1801-1272

\section{REFERENCES}

1. Aleksic T, Chitnis MM, Perestenko OV, et al. Type 1 insulin-like growth factor receptor translocates to the nucleus of human tumor cells. Cancer Res. 2010;70(16):6412-6419. https://doi.org/10.1158/ 0008-5472.can-10-0052.

2. Sarfstein R, Pasmanik-Chor M, Yeheskel A, et al. Insulin-like growth factor-I receptor (IGF-IR) translocates to nucleus and autoregulates IGF-IR gene expression in breast cancer cells. J Biol Chem. 2012;287 (4):2766-2776

3. Chitnis MM, Yuen JS, Protheroe AS, Pollak M, Macaulay VM. The type 1 insulin-like growth factor receptor pathway. Clin Cancer Res. 2008;14(20):6364-6370.

4. Aleksic T, Gray N, Wu X, et al. Nuclear IGF-1R interacts with regulatory regions of chromatin to promote RNA polymerase II recruitment 
and gene expression associated with advanced tumor stage. Cancer Research. 2018;78(13):3497-3509.

5. Sarfstein R, Werner $\mathrm{H}$. Minireview: nuclear insulin and insulin-like growth factor-1 receptors: a novel paradigm in signal transduction. Endocrinology. 2013;154(5):1672-1679.

6. Sehat B, Tofigh A, Lin Y, et al. SUMOylation mediates the nuclear translocation and signaling of the IGF-1 receptor. Sci Signal. 2010;3 (108):ra10-ra10.

7. Lin Y, Liu H, Waraky A, et al. SUMO-modified insulin-like growth factor 1 receptor (IGF-1R) increases cell cycle progression and cell proliferation. J Cell Physiol. 2017;232(10):2722-2730.

8. Deng $\mathrm{H}$, Lin $\mathrm{Y}$, Badin $\mathrm{M}$, et al. Over-accumulation of nuclear IGF-1 receptor in tumor cells requires elevated expression of the receptor and the SUMO-conjugating enzyme Ubc9. Biochem Biophys Res Commun. 2011;404(2):667-671.

9. Favre N, Camps M, Arod C, Chabert C, Rommel C, Pasquali C. Chemokine receptor CCR2 undergoes transportin1-dependent nuclear translocation. Proteomics. 2008;8(21):4560-4576.

10. Warsito D, Sjöström S, Andersson S, Larsson O, Sehat B. Nuclear IGF1R is a transcriptional co-activator of LEF1/TCF. EMBO Rep. 2012;13(3):244-250.

11. Wang SE. The functional crosstalk between HER2 tyrosine kinase and TGF- $\beta$ signaling in breast cancer malignancy. Journal of Signal Transduction. 2011;2011:1-8.
12. Dunn SE, Ehrlich M, Sharp NJ, et al. A dominant negative mutant of the insulin-like growth factor-I receptor inhibits the adhesion, invasion, and metastasis of breast cancer. Cancer Research. 1998;58(15): 3353-3361.

13. Schedlich LJ, Young TF, Firth SM, Baxter RC. Insulin-like growth factor-binding protein (IGFBP)-3 and IGFBP-5 share a common nuclear transport pathway in T47D human breast carcinoma cells. J Biol Chem. 1998;273(29):18347-18352.

14. Wu JD, Haugk K, Woodke L, Nelson P, Coleman I, Plymate SR. Interaction of IGF signaling and the androgen receptor in prostate cancer progression. J Cell Biochem. 2006;99(2):392-401.

15. Xiong $L$, Kou F, Yang $Y, W u$ J. A novel role for IGF-1R in p53-mediated apoptosis through translational modulation of the p53-Mdm2 feedback loop. J Cell Biol. 2007;178(6):995-1007.

How to cite this article: Chughtai S. The nuclear translocation of insulin-like growth factor receptor and its significance in cancer cell survival. Cell Biochem Funct. 2020;38:347-351. https://doi.org/10.1002/cbf.3479 